\section{Eye see}

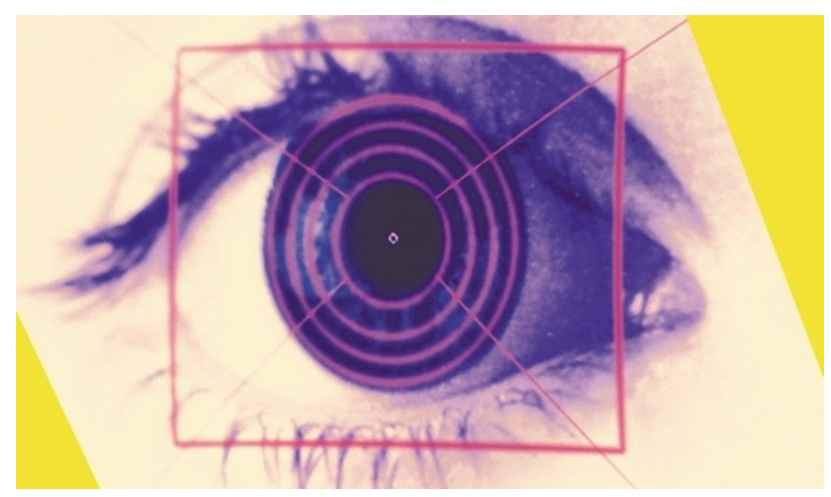

In some forms of retinal degenerative disease, including agerelated macular degeneration, the death of retinal cells can be due to defects in the underlying retinal pigment epithelial (RPE) cells. In animal models of these disorders, such as the Royal College of Surgeons (RCS) rat, progressive photoreceptor loss can be limited by transplantation of RPE cells into the subretinal space. A new study by Coffey et al., published in Nature Neuroscience, shows that such treatment can also lead to long-term preservation of cortical visual processing in these rats.

The photoreceptors of RCS rats die during the first three weeks after birth. Coffey and colleagues implanted cells from an immortalized human RPE cell line into the three-week-old rats, then monitored their visual ability and the responses of neurons in their visual cortex, to assess the effects of the gradual degeneration of photoreceptors and implantation of the RPE cells on central processing mechanisms.

Several weeks after implantation, the treated rats were as good as controls at tracking moving stripes and distinguishing between stripe patterns with different spatial frequencies, whereas untreated RCS rats were severely impaired. When the authors recorded from neurons in the primary visual cortex of the treated rats at seven months of age - by which time the corresponding neurons in untreated RCS rats are completely unresponsive to visual input they found that the neurons responded almost as well as those of controls with no visual impairment, with some neurons in treated rats showing physiological properties within the normal range.

When the eyes of the grafted animals were examined, they had around half as many photoreceptors as normal rats. It is interesting that these rats were able to perform at similar levels to normal rats despite having only partial protection against photoreceptor loss, indicating that the visual system can adapt to this kind of damage. These results are encouraging for the possible future use of implanted RPE cells in humans with degenerative retinal diseases, particularly as the use of immortalized cell lines would mean that a constant supply of cells for implantation could be maintained, avoiding the logistical and ethical difficulties associated with the use of fetal tissue for transplantation.

Rachel Jones

(2) References and links

ORIGINAL RESEARCH PAPER Coffey, P. J. et al. Long-term preservation of cortically dependent visual function in RCS rats by transplantation. Nature Neurosci. 19 December $2001(10.1038 / \mathrm{nn} 782)$

WEB SITES

WEB SITES
R. Lund's lab: http://insight.med.utah.edu/research/basic_science/rlund/rlund_lab.htm

\title{
A question of grooming
}

If you own a cat, you'll know how much time they spend preening themselves. Rather than a bad case of narcissism, this represents a type of grooming behaviour, which is essential to the well-being of most animals. But, like most things, it can go wrong. In humans, defective grooming behaviour ranges from the mild, such as nail-biting, to the severe, such as the obsessive-compulsive disorder trichotillomania (compulsive hair pulling that results in hair loss). Little is known about the genetics that underlie grooming behaviours, but Greer and Capecchi now report that mice with null mutations in the homeobox gene Hoxb8 groom excessively, potentially providing researchers with a valuable model for studying abnormal grooming behaviour in humans.

The authors constructed a null mutation in Hoxb8 by inserting a frameshift mutation in exon 1 and a neo gene, surrounded by loxP elements, in exon 2. They then removed the neo gene by inducing Cre-mediated recombination, to produce an allele with nonsense codons in both exons. The removal of neo turned out to be very important because when neo was retained, a skeletal phenotype was seen in homozygous mutant mice. This was most likely because the presence of neo causes the aberrant expression of nearby genes in the Hoxb cluster.

In the absence of neo, bald patches (sometimes accompanied by skin lesions) were the only other phenotype in $\mathrm{Hoxb8}^{-1}$ mice. There was no obvious skin defect to account for this, so the authors videoed the mutant mice for $24 \mathrm{~h}$ to see whether they showed any behavioural abnormalities. Strikingly, they found that the mutants spend twice as long grooming themselves as their wild-type littermates. This excessive licking and biting leads to the loss of body hair and to skin lesions. Furthermore, the mutants also excessively groom normal mice in the same cage, indicating that the behaviour is not the result of some abnormality in the skin of the mutant mice - rather it is a defect in grooming behaviour itself.

Greer and Capecchi also studied the expression of $H o x b 8$ and found high levels of expression in several parts of the brain that had been previously implicated in grooming behaviour in several mammals. Imaging studies have also detected abnormalities in the same brain regions in patients with obsessive-compulsive disorder, which fits with the similarity in phenotype between trichotillomania and the Hoxb8 mutants. This work therefore provides a valuable way into the biology that underlies grooming behaviour. The search will now be on to see whether variation in HOXB8 itself accounts for any of the variation in grooming behaviour in humans.

Mark Patterson

Editor, Nature Reviews Genetics

(2) References and links ORIGINAL RESEARCH PAPER Greer, J. M. \& Capecchi, M. R. Hoxb8 is required for normal grooming behavior in mice. Neuron 33, 23-34 (2002)

WEB SITES

Trichotillomania Learning Center: http://www.trich.org/ Mario Capecchi's lab: http://www.genetics.utah. edu/section2/sc205frm.html

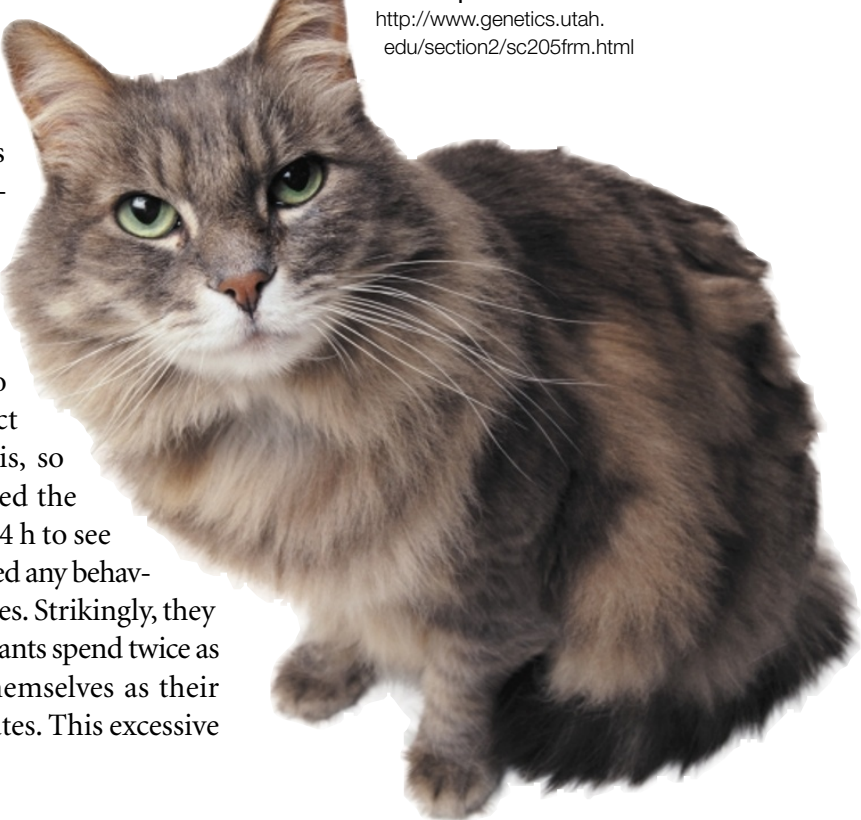

